\title{
Iterative Method to Compute the Maximal Concentration Slepian Band-limited Eigenfunction on the Sphere
}

\author{
Zubair Khalid and Rodney A. Kennedy \\ Research School of Engineering, \\ College of Engineering and Computer Science, \\ The Australian National University, \\ Canberra, ACT 0200, Australia \\ Email: \{zubair.khalid,rodney.kennedy\}@anu.edu.au
}

\begin{abstract}
The Slepian concentration problem on the sphere to maximize the energy concentration of a band-limited (in spherical harmonic degree) function is formulated as an eigenvalue problem, the solution of which gives rise to a family of band-limited eigenfunctions with optimal energy concentration in the spatial region on the sphere. In the family of band-limited eigenfunctions, the most concentrated (in the spatial region) eigenfunction is used for spatial windowing and spatial smoothing. We develop an iterative method to accurately compute the most concentrated band-limited Slepian eigenfunction for a given band-limit and a spatial region of interest. Taking into account the computational issues around the proposed iterative method, we also present the procedure for the practical implementation of the proposed method. In comparison to the computation of most concentration eigenfunction by the eigenvalue decomposition which gives a family of eigenfunction, the proposed method is computationally feasible even for large band-limits. Through examples, we also show that the proposed method attains sufficient numerical accuracy.
\end{abstract}

Index Terms-Concentration problem, spherical harmonics, eigenfunctions, 2-sphere (unit sphere).

\section{INTRODUCTION}

The development of spherical signal processing techniques finds applications in many diverse fields of science and engineering, such as geophysics [1], cosmology [2], quantum chemistry [3], acoustics [4] and wireless communication [5]. The extension of well formulated signal processing techniques - formulated in the Euclidean domain such as convolution, estimation, prediction, and filtering - to the spherical domain is a natural way to analyze the signal in these applications, where the signals and data-sets are inherently defined on the sphere. The Slepian concentration problem (formulated in Euclidean domain [6], [7]) has been extended and rigorously investigated for signals on the sphere [1], [8]. The Slepian concentration problem seeks band-limited (or space-limited) functions with optimal energy concentration in the spatial (or spectral) region. The solution of the Slepian concentration problem, formulated as an eigenvalue problem, provides a family of orthonormal eigenfunctions, referred to as Slepian eigenfunctions or the Slepian basis, which are optimally concentrated in both spatial and spectral domains. Slepian eigenfunctions have been used for localized spectral analysis [9] and spectral estimation [10]. Furthermore, exploiting the fact the most concentrated eigenfunction obtained from the Slepian concentration problem attains the lower bound imposed by the uncertainty principle on the simultaneous concentration of a signal in both spatial and spectral domains [9], [11], [12], the most concentrated band-limited eigenfunction has been used in obtaining the spatially localized spherical harmonic transform [11], [13] and proposed for smoothing of signals on the sphere [14].

In general, the computation of Slepian eigenfunctions require the quadrature to be evaluated over the spatially localized region [1], which cannot be computed accurately using a finite number of samples on the sphere. Therefore, the Slepian eigenfunctions can only be computed approximately. In addition, the computational complexity to determine Slepian eigenfunctions is large and scales with the band-limit, which causes the numerical computation to become infeasible for large bandlimits. For applications that only require the computation of most concentrated eigenfunction, it is clearly advantageous to develop alternative methods to compute the most concentrated eigenfunction rather than computing all of the eigenfunctions.

In this context, we develop an iterative method to compute the most concentrated (in a given spatial region of interest) band-limited Slepian eigenfunction. For the proposed method, we also present the procedure for the practical implementation that takes into account the computational issues around the proposed method. We show that the proposed method to compute most concentrated band-limited eigenfunction has less computational complexity as compared to the computation of all of the eigenfunctions. Furthermore, we also analyze the numerical accuracy achieved by the use of proposed method.

The rest of the paper is organized as follows. We present mathematical background for signals on the sphere and spherical harmonics in Section II. In Section III, we briefly review the formulation and applications of the Slepian concentration problem on the sphere and identify the research problem 
considered in this work. The proposed iterative method along with its implementation in practice is presented in Section IV, where we also carry out computational complexity analysis and numerical accuracy analysis of the proposed method. Finally, Section V concludes the paper.

\section{SignAls ON THE SPHERE}

\section{A. Sphere (Spherical Domain)}

The spherical domain, also referred as sphere or 2-sphere or unit sphere, is denoted by $\mathbb{S}^{2}$ and is defined as $\mathbb{S}^{2} \triangleq\{\boldsymbol{x} \in$ $\left.\mathbb{R}^{3}:|\boldsymbol{x}|=1\right\} \subset \mathbb{R}^{3}$, where $|\cdot|$ represents Euclidean norm. A point on $\mathbb{S}^{2}$ is given by a unit vector $\hat{\boldsymbol{x}} \equiv \hat{\boldsymbol{x}}(\theta, \phi) \triangleq$ $(\sin \theta \cos \phi, \sin \theta \sin \phi, \cos \theta)^{\prime} \in \mathbb{R}^{3}$, where $(\cdot)^{\prime}$ denotes the vector transpose operation, $\theta \in[0, \pi]$ is the co-latitude that is measured with respect to the positive $z$-axis and $\phi \in[0,2 \pi)$ is the longitude which is measured with respect to the positive $x$-axis in the $x-y$ plane.

\section{B. Signals on Sphere}

We consider the complex-valued square-integrable functions defined on the sphere. The set of such functions form a Hilbert space denoted by $L^{2}\left(\mathbb{S}^{2}\right)$ equipped with the inner product given by

$$
\langle f, h\rangle \triangleq \int_{\mathbb{S}^{2}} f(\hat{\boldsymbol{x}}) \overline{h(\hat{\boldsymbol{x}})} d s(\hat{\boldsymbol{x}}),
$$

for two functions $f$ and $h$ defined on $\mathbb{S}^{2}$. Here $d s(\hat{\boldsymbol{x}})=$ $\sin \theta d \theta d \phi$ is the differential area element on $\mathbb{S}^{2}$. The inner product induces a norm $\|f\| \triangleq\langle f, f\rangle^{1 / 2}$. We refer the functions with finite energy (finite induced norm) as "signals on the sphere". We also define $\langle f, g\rangle_{R} \triangleq \int_{R} f(\hat{\boldsymbol{x}}) \overline{g(\hat{\boldsymbol{x}})} d s(\hat{\boldsymbol{x}})$ and $\|f\|_{R} \triangleq\langle f, f\rangle_{R}^{1 / 2}$ as the energy of the signal $f$ with in the region $R$.

\section{Operator on the Sphere}

Using general Fredholm integral equation, we define an operator $\mathcal{S}$ for signals on the sphere as [15]

$$
(\mathcal{S} f)(\hat{\boldsymbol{x}})=\int_{\mathbb{S}^{2}} S(\hat{\boldsymbol{x}}, \hat{\boldsymbol{y}}) f(\hat{\boldsymbol{y}}) d s(\hat{\boldsymbol{y}}),
$$

where $S(\hat{\boldsymbol{x}}, \hat{\boldsymbol{y}})$ is the kernel for an operator $\mathcal{S}$.

\section{Spherical Harmonics and Spectral (Fourier) Domain}

Spherical harmonic functions form a complete orthonormal set of basis functions for $L^{2}\left(\mathbb{S}^{2}\right)$. The spherical harmonic function (or spherical harmonic for short) $Y_{\ell}^{m}(\theta, \phi)$ for integer degree $\ell \geq 0$ and integer order $|m| \leq \ell$ is defined as [15]

$$
Y_{\ell}^{m}(\theta, \phi)=\sqrt{\frac{2 \ell+1}{4 \pi} \frac{(\ell-m) !}{(\ell+m) !}} P_{\ell}^{m}(\cos \theta) e^{i m \phi},
$$

where $P_{\ell}^{m}$ denotes the associated Legendre function of integer degree $\ell$ and integer order $m$ and is defined as [15]

$$
\begin{aligned}
P_{\ell}^{m}(u) & =\frac{(-1)^{m}}{2^{\ell} \ell !}\left(1-u^{2}\right)^{m / 2} \frac{d^{\ell+m}}{d u^{\ell+m}}\left(u^{2}-1\right)^{\ell} \\
P_{\ell}^{-m}(u) & =(-1)^{m} \frac{(\ell-m) !}{(\ell+m) !} P_{\ell}^{m}(u),
\end{aligned}
$$

for $|u| \leq 1$.

By the completeness of spherical harmonics, we can expand any signal $f \in L^{2}\left(\mathbb{S}^{2}\right)$ as

$$
f(\hat{\boldsymbol{x}})=\sum_{\ell=0}^{\infty} \sum_{m=-\ell}^{\ell}(f)_{\ell}^{m} Y_{\ell}^{m}(\hat{\boldsymbol{x}}),
$$

where equality is understood in the sense of convergence in the mean and

$$
(f)_{\ell}^{m} \triangleq\left\langle f, Y_{\ell}^{m}\right\rangle=\int_{\mathbb{S}^{2}} f(\hat{\boldsymbol{u}}) \overline{Y_{\ell}^{m}(\hat{\boldsymbol{u}})} d s(\hat{\boldsymbol{u}})
$$

denotes the spherical harmonic (Fourier) coefficient of degree $\ell$ and order $m$. The spherical harmonic coefficients $(f)_{\ell}^{m}$ form the spectral domain representation of a signal. The signal $f \in$ $L^{2}\left(\mathbb{S}^{2}\right)$ is defined to be band-limited at degree $L$ if $(f)_{\ell}^{m}=$ 0 for $\ell \geq L$. The set of bandlimited signals forms an $L^{2}$ dimensional subspace of $L^{2}\left(\mathbb{S}^{2}\right)$, which is denoted by $\mathcal{H}_{L}$.

Let the column vector $\mathbf{f}$ of size $L^{2}$, given by

$$
\mathbf{f}=\left((f)_{0}^{0},(f)_{1}^{-1},(f)_{1}^{0},(f)_{1}^{1},(f)_{2}^{-2}, \cdots,(f)_{L}^{L}\right)^{\prime},
$$

contains all spherical harmonic coefficients $(f)_{\ell}^{m}$ of a bandlimited signal $f \in \mathcal{H}_{L}$ and represents the spectral domain representation of the signal.

\section{PRoblem Formulation}

\section{A. Slepian Concentration Problem on the Sphere}

The concentration problem on the sphere for finding the functions with simultaneous concentration in both spatial and spectral domains has been extensively investigated [1], [8]. In order to maximize the spatial concentration of a bandlimited signal $f \in \mathcal{H}_{L}$ within the spatial region $R \subset \mathbb{S}^{2}$, we seek to maximize the spatial concentration (energy) ratio $\lambda$ given by [1],

$$
\lambda=\frac{\|f\|_{R}^{2}}{\|f\|^{2}}, \quad 0<\lambda<1 .
$$

which can be equivalently expressed in spectral domain as

$$
\lambda=\frac{\sum_{\ell=0}^{L} \sum_{m=-\ell}^{\ell} \sum_{\ell^{\prime}=0}^{L} \sum_{m^{\prime}=-\ell^{\prime}}^{\ell^{\prime}}(f)_{\ell}^{m} \overline{(f)_{\ell^{\prime}}^{m^{\prime}}} E_{\ell \ell^{\prime}, m m^{\prime}}}{\sum_{\ell=0}^{L} \sum_{m=-\ell}^{\ell}(f)_{\ell}^{m} \overline{(f)_{\ell}^{m}}},
$$

where

$$
E_{\ell \ell^{\prime}, m m^{\prime}}=\int_{R} Y_{\ell}^{m}(\hat{\boldsymbol{x}}) \overline{Y_{\ell^{\prime}}^{m^{\prime}(\hat{\boldsymbol{x}})}} d s(\hat{\boldsymbol{x}}) .
$$

By defining a matrix $\mathbf{E}$ of size $L^{2} \times L^{2}$ with entries $E_{\ell \ell^{\prime}, m m^{\prime}}$ such that the same ordering as in $\mathbf{f}$ is used for indexing of rows (identified by $\ell, m$ ) and columns (identified by $\ell^{\prime}, m^{\prime}$ ), the concentration ratio in (7) can be compactly written as

$$
\lambda=\frac{\mathbf{f}^{H} \mathbf{E f}}{\mathbf{f}^{H} \mathbf{f}},
$$

where the superscript $H$ denotes Hermitian operation. The problem to maximize the concentration ratio in (9) can be solved as an algebraic eigenvalue problem

$$
\mathbf{E f}=\lambda \mathbf{f},
$$


the solution of which gives $L^{2}$ orthonoromal eigenfunctions. The eigenvalue $0<\lambda<1$ associated with each bandlimited eigenfunction serve as a measure of the concentration of the eigenfunction in the region $R$. Let the eigenfunctions be denoted by $f_{p}, p \in\left[1,2, \ldots, L^{2}\right]$ and the associated eigenvalue for each eigenfunction is denoted by $\lambda_{p}$, where we index the eigenfunctions such that $1 \geq \lambda_{1} \geq \lambda_{2} \geq \cdots \geq \lambda_{L^{2}} \geq 0$.

\section{B. Use of Concentrated Slepian Eigenfunction}

The solution of Slepian concentration problem provides spatially concentrated and band-limited eigenfunctions. Due to the reason that the most concentrated band-limited eigenfunction attains the lower bound imposed by the uncertainty principle on the simultaneous concentration of the function in both spatial and spectral domains, the most concentrated eigenfunction is of great use in applications. We briefly review the use of eigenfunction with maximal spatial concentration in azimuthally symmetric and elliptical regions on the sphere.

\section{Concentration in Azimuthally Symmetric Region}

For a band-limited eigenfunction with the band-limit $L$ and maximal spatial concentration in an azimuthally symmetric polar (North pole) cap region $R_{\left(\theta_{\mathrm{c}}\right)} \subset \mathbb{S}^{2}$, given by

$$
R_{\left(\theta_{\mathrm{c}}\right)} \triangleq\left\{(\theta, \phi): \theta \leq \theta_{\mathrm{c}}\right\}
$$

where $\theta_{\mathrm{c}}$ and the band-limit $L$ are related by

$$
\theta_{\mathrm{c}}=\frac{2 \pi}{L+1},
$$

it is established that one eigenfunction is at least $99 \%$ concentrated [9], [11]. Therefore, using (12) we have a one parameter maximally band-limited eigenfunction with concentration of $99 \%$ in the polar cap region $R$. Such an eigenfunction has been used for obtaining spatially localized spherical harmonic representation of the signal. Furthermore, it has also been proposed as a suitable candidate for spatial smoothing (azimuthally symmetric convolution [16]) of signals on the sphere [14].

Example 1: As an example, we obtain the most concentrated band-limited eigenfunction $f_{1}$ using the formulation of Slepian concentration problem in (10) for $L=17$ and azimuthally symmetric spatial region $R_{\left(\theta_{\mathrm{c}}\right)}$ with $\theta_{\mathrm{c}}$ given in (12). The eigenfunction $f_{1}$ with spatial concentration $\lambda_{1}=0.9944$ in the region $R_{\left(\theta_{\mathrm{c}}\right)}$ is plotted in Fig. 1(a) on the sphere and its spectral domain representation formed by spherical harmonic coefficients, $\left(f_{1}\right)_{\ell}^{m}$, is shown in Fig. $1(\mathrm{~b})$. We note that the eigenfunction, $f_{1}(\theta, \phi)=f_{1}(\theta)$, is azimuthally symmetric and therefore all non-zero order spherical harmonic coefficients $\left(f_{1}\right)_{\ell}^{m}$ are zero, that is, $\left(f_{1}\right)_{\ell}^{m}=0$ for $m \neq 0$.

\section{Concentration in Elliptical Region}

A band-limited eigenfunction with minimum band-limit and maximal spatial concentration in the elliptical region on the sphere has been used as a window function in obtaining directional spatially localized spherical harmonic transform [13].
We parameterize the elliptical region using the focus colatitude $\theta_{\mathrm{c}}$ of the elliptical region along the positive $x$-axis and the arc length $a$ of the semi-major axis [13]:

$$
\begin{aligned}
R_{\left(\theta_{\mathrm{c}}, a\right)} \triangleq & \left\{(\theta, \phi): \triangle_{s}\left((\theta, \phi),\left(\theta_{\mathrm{c}}, 0\right)\right)\right. \\
& \left.+\triangle_{s}\left((\theta, \phi),\left(\theta_{\mathrm{c}}, \pi\right)\right) \leq 2 a\right\},
\end{aligned}
$$

where $0 \leq \theta_{\mathrm{c}} \leq a \leq \pi / 2$. Here $\triangle_{s}\left((\theta, \phi),\left(\theta^{\prime}, \phi^{\prime}\right)\right)=$ $\arccos \left(\sin \theta \sin \theta^{\prime} \cos \left(\phi-\phi^{\prime}\right)+\cos \theta \cos \theta^{\prime}\right)$ denotes the angular distance between the two points parameterized by $(\theta, \phi)$ and $\left(\theta^{\prime}, \phi^{\prime}\right)$ on the sphere.

The eigenfunction with minimum band-limit and maximal spatial concentration is determined by finding the minimum value of the band-limit $L$ which ensures that $\lambda_{1}$ is greater than or equal to the desired energy concentration (90\% or $99 \%)$. Thus, the band-limit $L$, focus of an elliptical region $\theta_{c}$ and the arc length $a$ of the semi-major axis fully parameterize the eigenfunction.

Example 2: As an illustration, we obtain the eigenfunction $f_{1}$ for band-limit $L=17$ and spatial concentration in the elliptical region $\mathcal{R}_{\left(\theta_{\mathrm{c}}, a\right)}$ with focus $\theta_{\mathrm{c}}=\pi / 6$ and $a=\pi / 6+$ $\pi / 60$. The eigenfunction $f_{1}$ with spatial concentration of $\lambda_{1}=$ 0.9919 in the region $\mathcal{R}_{\left(\theta_{\mathrm{c}}, a\right)}$ is shown in the spatial (sphere) domain in Fig. 2(a) and in the spectral domain in Fig. 2(b).

\section{Research Problem under Consideration}

The most concentrated eigenfunction for the given bandlimit $L$ and the spatial region $R$ is obtained by numerically solving an algebraic eigenvalue problem in (10). Such computation of the most concentrated eigenfunction requires the eigenvalue decomposition of the matrix $\mathbf{E}$ which becomes infeasible and inaccurate due to the following reasons: 1) the entries of the matrix $\mathbf{E}$ given in (8) may not be computed accurately for an arbitrary region $R$, and 2) the computational complexity to carry out eigenvalue decomposition naively is of the order $O\left(\left(L^{2}\right)^{3}\right)=O\left(L^{6}\right)$, which is computationally intensive and becomes infeasible for large band-limit $L$. However, the complexity can be reduced to $O\left(\left(L^{2}\right)^{2.376}\right)$ by employing efficient algorithms for matrix multiplications and eigenvalue decomposition [17]. In applications where we only require one eigenfunction (most concentrated), it is sensible and advantageous to seek alternative methods to accurately compute eigenfunction with reduced computational complexity rather than the eigenvalue decomposition which computes $L^{2}$ eigenfunctions.

In this work, we propose an iterative method to accurately (not exactly) compute the maximally concentrated bandlimited eigenfunction. In comparison, the proposed method has much reduced complexity. We also show, through numerical experiments, that the sufficient accuracy is attained by the proposed method.

We again emphasize that the computation of eigenfunction through the solution of eigenvalue problem in (10) is also approximate for an arbitrary spatial region $R$ as the matrix $\mathbf{E}$ can only be computed approximately for an arbitrary region. However, if the region $R$ is azimuthally symmetric as given in (11), the entries of the matrix $\mathbf{E}$ given in (8) can be analytically 


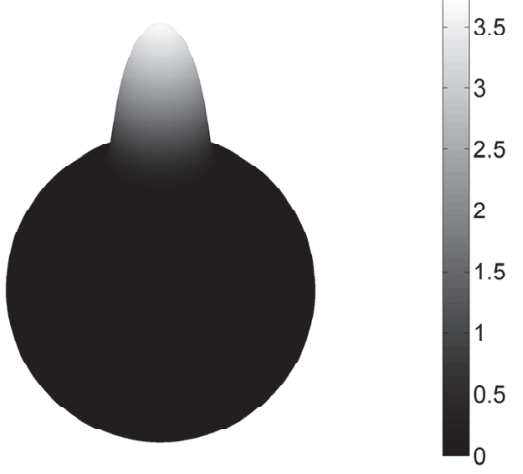

(a)

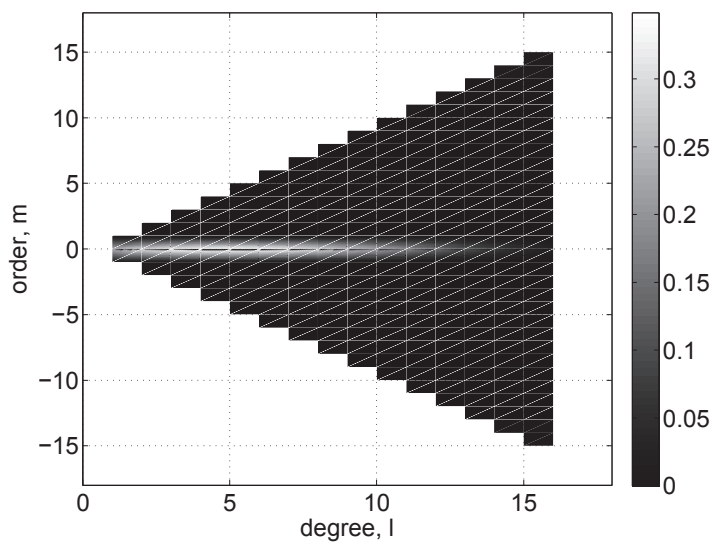

(b)

Fig. 1: The most concentrated band-limited eigenfunction $f_{1}$ obtained from the solution Slepian concentration problem in (10) for band-limit $L=17$ and azimuthally symmetric spatial region $R_{\left(\theta_{\mathrm{c}}\right)}$ with $\theta_{\mathrm{c}}$ given in (12). The eigenfunction $f_{1}$ in (a) spatial domain (on the sphere) and (b) spectral domain as $\left(f_{1}\right)_{\ell}^{m}$. Since the eigenfunction $f_{1}$ is azimuthally symmetric, all non-zero order spherical harmonic coefficients $\left(f_{1}\right)_{\ell}^{m}$ are zero.

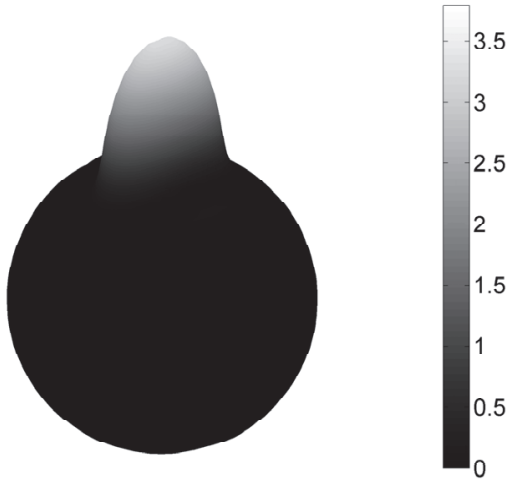

(a)

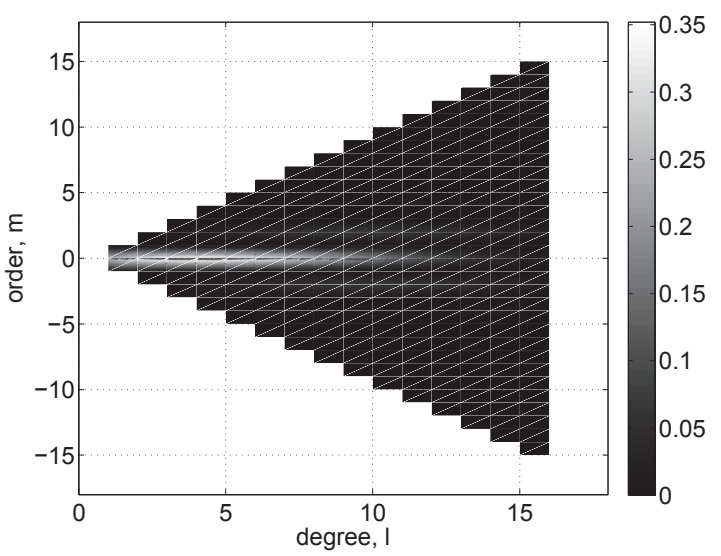

(b)

Fig. 2: The most concentrated band-limited eigenfunction $f_{1}$ obtained from the solution Slepian concentration problem in (10) for band-limit $L=17$ and spatial concentration in the elliptical region $\mathcal{R}_{\left(\theta_{\mathrm{c}}, a\right)}$ with focus $\theta_{\mathrm{c}}=\pi / 6$ and $a=\pi / 6+\pi / 60$. The eigenfunction $f_{1}$ in (a) spatial domain (on the sphere) and (b) spectral domain as $\left(f_{1}\right)_{\ell}^{m}$.

computed. Furthermore, for the azimuthally symmetric region, the eigenfunctions can also be determined by the eigenvalue decomposition of the matrix $\mathbf{G}$ (with simple analytic entries) that commutes with $\mathbf{E}$, that is, $\mathbf{E G}=\mathbf{G E}$ [1], [9].

\section{ITERATIVE COMPUTATION OF SLEPIAN EIGENFUNCTION}

We present an iterative method to compute the maximally spatially concentrated band-limited eigenfunction for a given band-limit $L$ and a spatial region $R$. We note that the bandlimit $L$ and spatial region $R$ fully characterize the Slepian concentration problem. We first define the selection operators [15] on the sphere, following the operator action in (2).

Definition 1 (Spatial Selection Operator): Define the spatial selection operator $\mathcal{S}_{R}$ which selects the function in a spatial region $R \subset \mathbb{S}^{2}$ with kernel given by

$$
S_{R}(\hat{\boldsymbol{x}}, \hat{\boldsymbol{y}}) \triangleq I_{R}(\hat{\boldsymbol{x}}) \delta(\hat{\boldsymbol{x}}, \hat{\boldsymbol{y}}),
$$

where $I_{R}(\hat{\boldsymbol{x}})=1$ for $\hat{\boldsymbol{x}} \in R \subset \mathbb{S}^{2}$ and $I_{R}(\hat{\boldsymbol{x}})=0$ for $\hat{\boldsymbol{x}} \in \mathbb{S}^{2} \backslash R$ is an indicator function of the spatial region $R$ and $\delta(\hat{\boldsymbol{x}}, \hat{\boldsymbol{y}})$ is a Dirac delta function on the sphere [15].

Definition 2 (Spectral Selection Operator): Define the spectral selection operator $\mathcal{S}_{L}$ which band-limits the signal at $L$ with kernel given by

$$
S_{L}(\hat{\boldsymbol{x}}, \hat{\boldsymbol{y}}) \triangleq \sum_{\ell=0}^{L-1} \sum_{m=-\ell}^{\ell} Y_{\ell}^{m}(\hat{\boldsymbol{x}}) \overline{Y_{\ell}^{m}(\hat{\boldsymbol{y}})} .
$$




\section{A. Proposed Iterative Method}

Here, an iterative method is presented to compute the maximally concentrated band-limited eigenfunction. For the given band-limit $L$ and the spatial region $R$, the following characteristics of the most concentrated eigenfunction $f_{1}$ has been observed for different spatial regions:

- $f_{1}(\hat{\boldsymbol{x}})$ is positive for $\hat{\boldsymbol{x}} \in R \subset \mathbb{S}^{2}$, and

- $f_{1}(\hat{\boldsymbol{x}})$ is monotonic over the spatial region $R$, that is, $f_{1}(\hat{\boldsymbol{x}})$ monotonically decreases towards the boundaries of the region $R$.

Although these characteristics of the most concentrated eigenfunction are not supported by theoretical evidence, the most concentrated eigenfunction for different values of the bandlimit $L$ and different spatial region exhibits these properties.

Let $g_{0} \in \mathcal{H}_{L}$ be the band-limited function such that the spatially localized function $\mathcal{S}_{R} g_{0}$ satisfies these properties. We propose that the function $g_{k} \in \mathcal{H}_{L}$ given by

$$
g_{k}=\frac{\mathcal{S}_{L} \mathcal{S}_{R} g_{k-1}}{\left\|\mathcal{S}_{L} \mathcal{S}_{R} g_{k-1}\right\|},
$$

for $k=1,2, \ldots$, converges to the most concentrated eigenfunction $f_{1}$ as $k \rightarrow \infty$. During each iteration, the proposed method increases the energy of a band-limited function in the spatial region $R$, that is, $\left\|g_{k}\right\|_{R} \geq\left\|g_{k-1}\right\|_{R}$, where the equality means that $g_{k}$ has maximum energy with in the spatial region. The presence of the term $\left\|\mathcal{S}_{L} \mathcal{S}_{R} g_{k-1}\right\|$ in (16) ensures that $g_{k}$ has unit energy after each iteration step, that is, $\left\|g_{K}\right\|=1$. Since we enforce $\left\|g_{K}\right\|=1$ at each iteration step, $0<\left\|g_{K}\right\|_{R}<1$ measures the spatial (energy) concentration of $g_{K}$ with in the spatial region $R$. One of the simplest choice for $g_{0} \in \mathcal{H}_{L}$ is $g_{0}(\hat{\boldsymbol{x}})=1$. Another possible choice to choose $g_{0} \in \mathcal{H}_{L}$ is to band-limit the indicator function $I(\hat{\boldsymbol{x}})$ of the region at degree $L$.

\section{B. Implementation in Practice}

The iterative computation of an eigenfunction using (16) requires the implementation of selection operators on the sphere. For the representation of a function on the sphere, it is necessary to adopt sampling scheme on the sphere to discretize the function. We consider a sampling scheme on the sphere that requires the optimal number $\left(L^{2}\right)$ of samples on the sphere for an accurate computation of spherical harmonic transform (SHT) of a signal band-limited at $L$ [18]. Let $\mathfrak{S}(L)$ denotes the sampling grid proposed in [18] on the sphere which consist of $L^{2}$ samples on the sphere.

The spatial selection operator $\mathcal{S}_{R}$ can be simply implemented as masking of the signal in the spatial domain using the indicator function $I_{R}(\hat{\boldsymbol{x}})$ of the spatial region $R$. Since $\mathcal{S}_{R} g_{k-1}$ is spatially localized, it is not band-limited and therefore, the spherical harmonic transform cannot be computed accurately in practice using the finite number of samples on the sphere due to the aliasing errors. Consequently, $\mathcal{S}_{R} g_{k-1}$ cannot be band-limited using the selection operator $\mathcal{S}_{L}$.

In order to implement the proposed iterative method, we use the high resolution spatial grid $\mathfrak{S}(M), M>L$ so that the error in computing spherical harmonic transform due to the aliasing is reduced. We later show, through numerical experiments, that $M=5 L$ is sufficient resolution of the grid to sample the signals during the implementation. Furthermore, since the energy of a band-limited function in the spatial region $R$ should be increased during each iteration, that is, $\left\|g_{k}\right\|_{R} \geq\left\|g_{k-1}\right\|_{R}$, we check this condition during each iteration and stop the procedure once $\left(\left\|g_{k}\right\|_{R}-\left\|g_{k-1}\right\|_{R}\right)<\epsilon$ for some small $\epsilon$. Using the property of an eigenfunction noted in [1] that $\left\|f_{1}\right\|_{R}=\left\|\mathcal{S}_{L} \mathcal{S}_{R} f_{1}\right\|$, we implement the condition $\left\|g_{k}\right\|_{R} \geq\left\|g_{k-1}\right\|_{R}$ as $\left\|\mathcal{S}_{L} \mathcal{S}_{R} g_{k}\right\| \geq\left\|\mathcal{S}_{L} \mathcal{S}_{R} g_{k-1}\right\|$ because it can be computed readily during implementation.

We here outline the procedure to implement the iterative computation of an approximate eigenfunction, formulated in (16), for a given band-limit $L$ and the spatial region $R$. Let $g_{K}$ denotes the function computed after $K$ iterations which is obtained using the procedure, Compute Eigenfunction, summarized below:

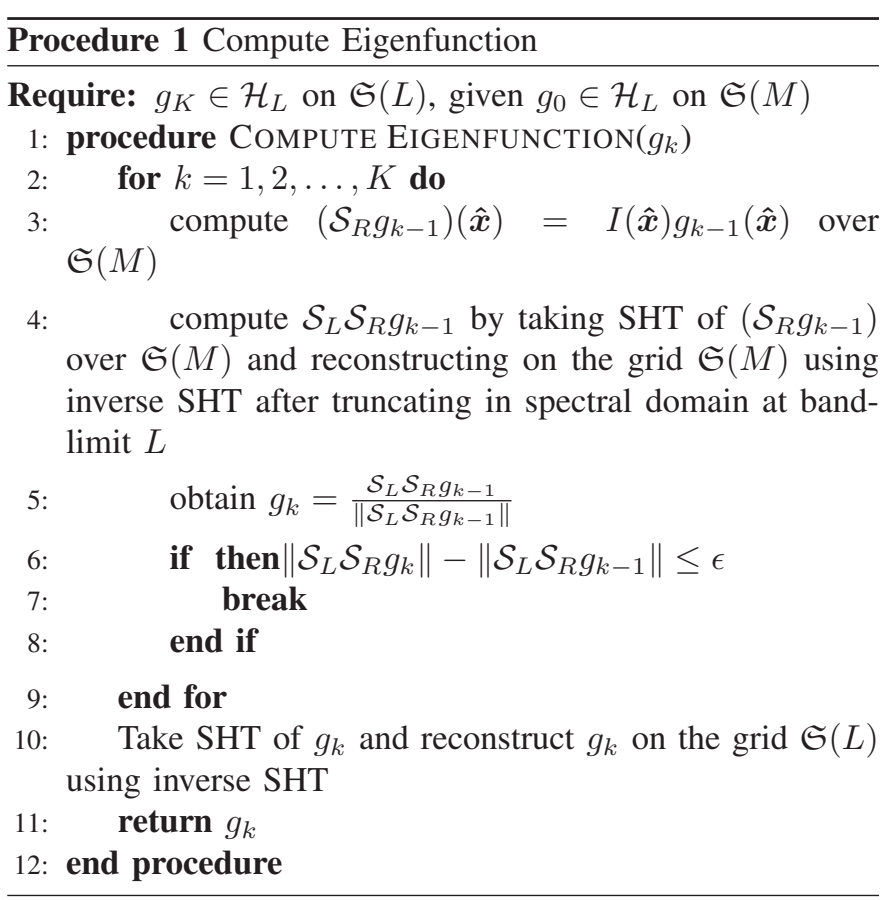

\section{Computational Complexity}

Now we analyse the computational complexity of the proposed iterative method to compute concentrated eigenfunction. The complexity of the proposed procedure scales with the band-limit $L$. The major computational burden during each iteration of the proposed method is due to spherical harmonic transform, which can be implemented with complexity $O\left(M^{3}\right) \equiv O\left(L^{3}\right)$ [18], [19]. For $K$ iterations, the complexity is scaled by $K$ and therefore the overall asymptotic complexity of the proposed method is $O\left(K L^{3}\right)$. In comparison to the computation of eigenfunction through eigenvalue decomposition, we note that the proposed method has much reduced complexity. 


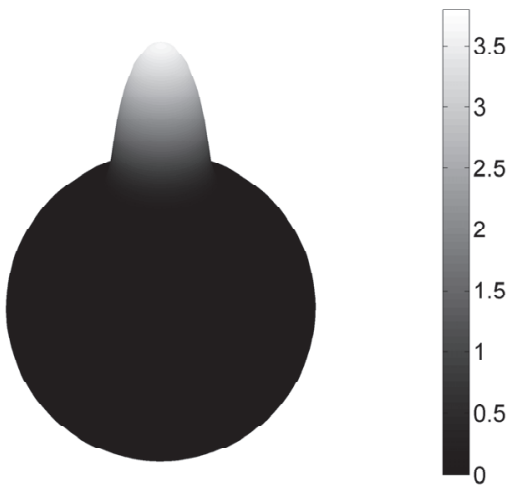

(a)

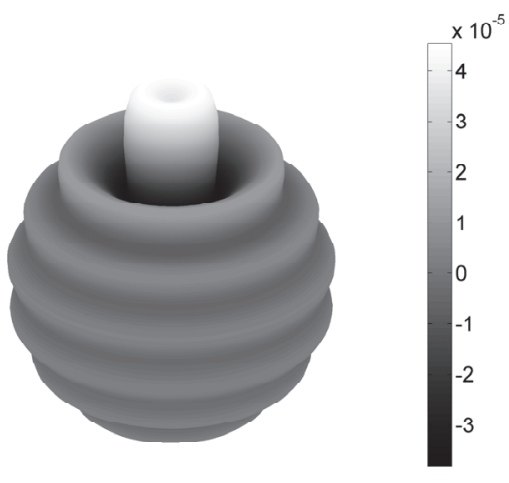

(c)

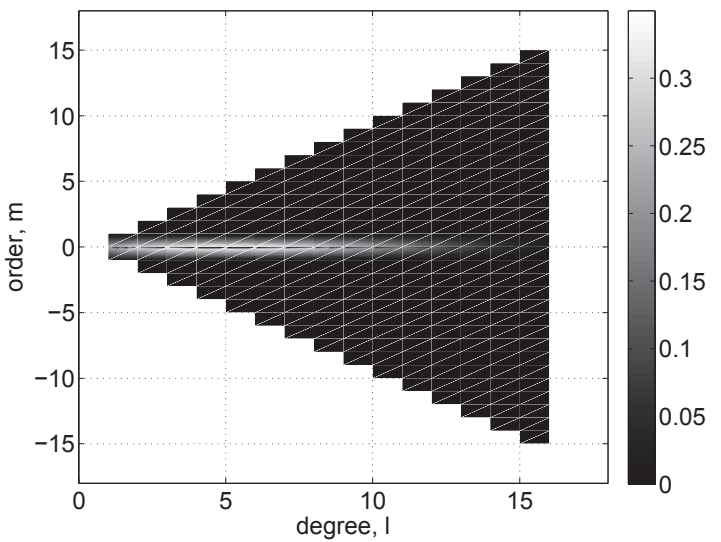

(b)

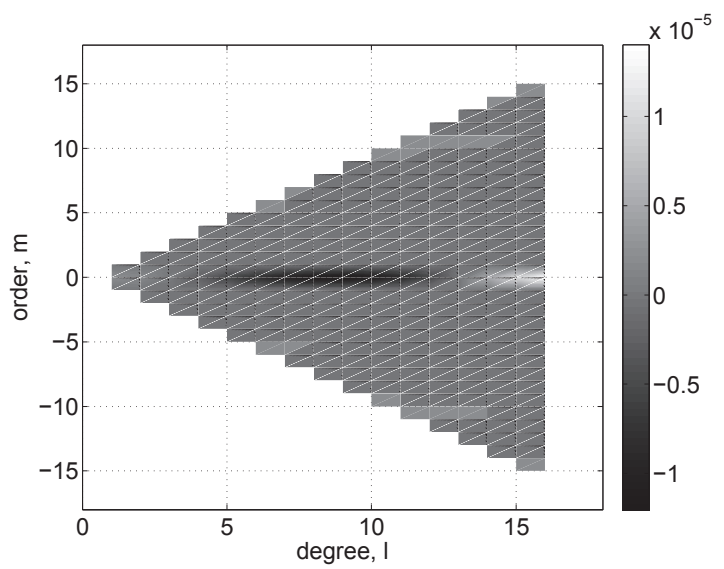

(d)

Fig. 3: An iteratively constructed eigenfunction $g_{K}$, with band-limit $L=17$, spatial concentration in an azimuthally symmetric spatial region $R_{\left(\theta_{\mathrm{c}}\right)}$ and $K=12$, in (a) spatial domain in and in (b) spectral domain as $\left(g_{K}\right)_{\ell}^{m}$. The difference between the iteratively constructed $g_{K}$ and $f_{1}$ (obtained from the Slepian concentration problem) in (c) spatial domain as $g_{K}(\hat{\boldsymbol{x}})-f_{1}(\hat{\boldsymbol{x}})$ and in (d) spectral domain as $\left(g_{K}\right)_{\ell}^{m}-\left(f_{1}\right)_{\ell}^{m}$.

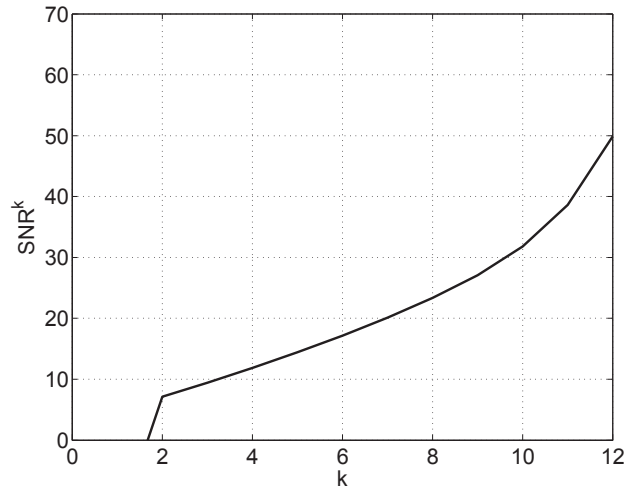

Fig. 4: The $\mathrm{SNR}^{k}$ after each iteration of the computation of $g_{k}$ with band-limit $L=17$ and spatial concentration in an azimuthally symmetric spatial region $R_{\left(\theta_{c}\right)}$.

\section{Numerical Accuracy Analysis}

We illustrate that the sufficient accuracy is attained by the proposed method. In order to quantify the level of accuracy, we define the signal-to-noise ratio (SNR) for the computed eigenfunction $g_{k}$ after $k$-th iteration as $g_{k}$ as

$$
\mathrm{SNR}^{k}=20 \log \frac{\left\|f_{1}\right\|}{\left\|f_{1}-g_{k}\right\|} .
$$

We revisit the concentration problems considered in Example 1 and Example 2 and iteratively construct the most concentrated eigenfunction using our proposed method. In our implementation, we choose $M=5 L$ and $\epsilon=10^{-3}$.

Example 1-Revisit: The parameters of the Slepian concentration problem are: band-limit $L=17$ and azimuthally symmetric spatial region $R_{\left(\theta_{\mathrm{c}}\right)}$ with $\theta_{\mathrm{c}}$ given in (12). We consider $g_{0}(\hat{\boldsymbol{x}})=1$ and iteratively compute the eigenfunction $g_{K}$. The procedure is stopped at $K=12$ when $\left\|\mathcal{S}_{L} \mathcal{S}_{R} g_{k}\right\|-$ $\left\|\mathcal{S}_{L} \mathcal{S}_{R} g_{k-1}\right\| \leq \epsilon$. The iteratively constructed eigenfunction $g_{K}$ with spatial concentration $\left\|g_{K}\right\|_{R}=0.9984$ is shown in spatial domain in Fig. 3(a) and in spectral domain in Fig. 3(b) as $\left(g_{K}\right)_{\ell}^{m}$. In order to further analyze the accuracy, we also plot the difference between $g_{K}$ and $f_{1}$ in the spatial domain 


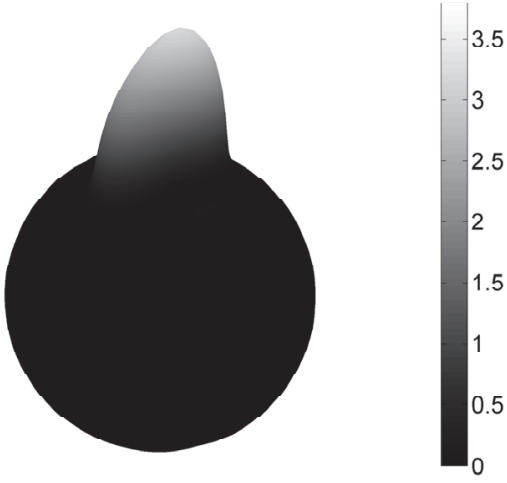

(a)

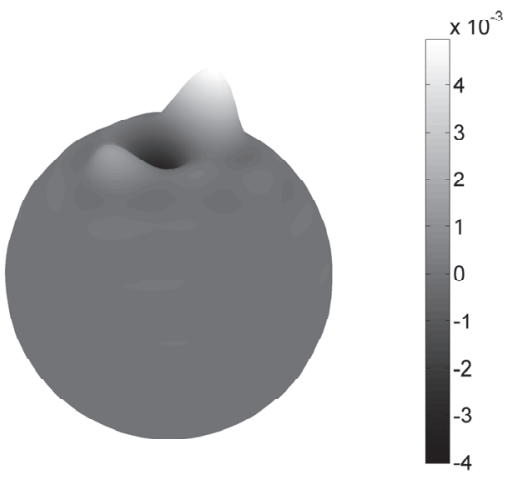

(c)

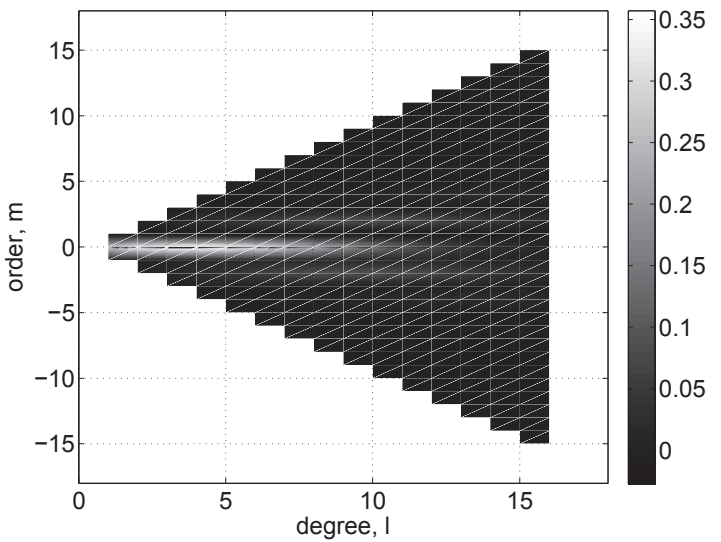

(b)

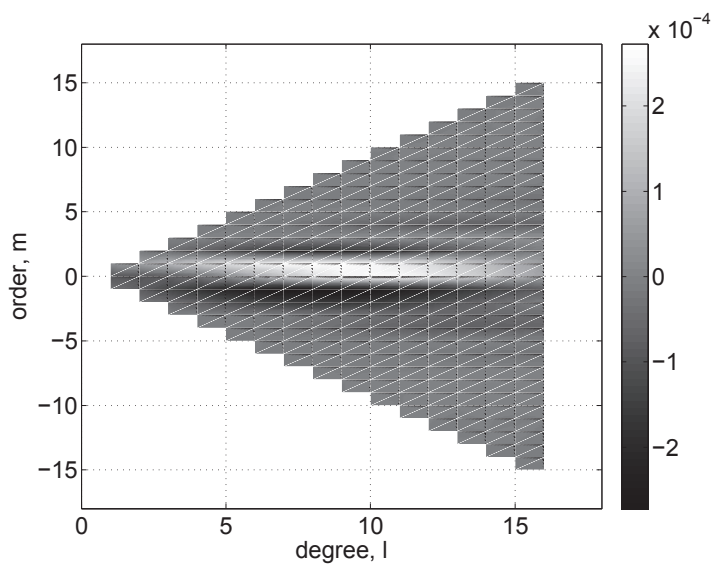

(d)

Fig. 5: An iteratively constructed eigenfunction $g_{K}$, with band-limit $L=17$, spatial concentration in an elliptical region $\mathcal{R}_{\left(\theta_{c}, a\right)}$ with focus $\theta_{\mathrm{c}}=\pi / 6$ and $a=\pi / 6+\pi / 60$ and $K=34$, in (a) spatial domain in and in (b) spectral domain as $\left(g_{K}\right)_{\ell}^{m}$. The difference between the iteratively constructed $g_{K}$ and $f_{1}$ (obtained from the Slepian concentration problem) in (c) spatial domain as $g_{K}(\hat{\boldsymbol{x}})-f_{1}(\hat{\boldsymbol{x}})$ and in (d) spectral domain as $\left(g_{K}\right)_{\ell}^{m}-\left(f_{1}\right)_{\ell}^{m}$.

in Fig. 3(c) as $g_{K}(\hat{\boldsymbol{x}})-f_{1}(\hat{\boldsymbol{x}})$ and in the spectral domain in Fig. 3(d) as $\left(g_{K}\right)_{\ell}^{m}-\left(f_{1}\right)_{\ell}^{m}$. The $\mathrm{SNR}^{k}$ after each iteration is shown in Fig. 4, where it can be observed that the $\mathrm{SNR}^{k}$ improves with the iteration step.

Example 2 - Revisit: The parameters of the Slepian concentration problem are: band-limit $L=17$ and elliptical spatial region $\mathcal{R}_{\left(\theta_{\mathrm{c}}, a\right)}$ with focus $\theta_{\mathrm{c}}=\pi / 6$ and $a=\pi / 6+\pi / 60$. We consider $g_{0}(\hat{\boldsymbol{x}})=1$ and iteratively compute the eigenfunction $g_{K}$. The procedure is stopped at $K=34$ as $\left\|\mathcal{S}_{L} \mathcal{S}_{R} g_{k}\right\|-$ $\left\|\mathcal{S}_{L} \mathcal{S}_{R} g_{k-1}\right\| \leq \epsilon$. The iteratively computed eigenfunction $g_{K}$ with spatial concentration $\left\|g_{K}\right\|_{R}=0.9933$ in the region $\mathcal{R}_{\left(\theta_{\mathrm{c}}, a\right)}$ is shown in the spatial domain in Fig. 5(a) and in the spectral domain in Fig. 5(b). We also plot the difference between $g_{K}$ and $f_{1}$ in the spatial domain in Fig. 5(c) as $g_{K}(\hat{\boldsymbol{x}})-f_{1}(\hat{\boldsymbol{x}})$ and in the spectral domain in Fig. 5(d) as $\left(g_{K}\right)_{\ell}^{m}-\left(f_{1}\right)_{\ell}^{m}$. The $\mathrm{SNR}^{k}$ after each iteration is shown in Fig. 6, demonstrating the improvement in $\mathrm{SNR}^{k}$ with the iteration step.

The improvement in SNR with the iteration step and the error between $g_{K}$ and $f_{1}$ in both examples indicate that the proposed iterative computation of the eigenfunction is sufficiently accurate.

\section{CONCLusions}

Among a family of Slepian eigenfunctions obtained as solutions of the Slepian concentration problem on the sphere, which seeks to maximize the concentration of a band-limited function in the spatial region of interest, the eigenfunction with maximum concentration in the spatial region is used in many applications for spatial windowing and spatial smoothing. We have developed an iterative method for the accurate computation of the most concentrated band-limited Slepian eigenfunction for a given band-limit and a spatial region of interest. In comparison to the computation of the most concentrated eigenfunction by solving an eigenvalue problem, we have shown that the proposed method has much reduced computational complexity. Furthermore, We have presented examples to obtain the most concentrated eigenfunction for 


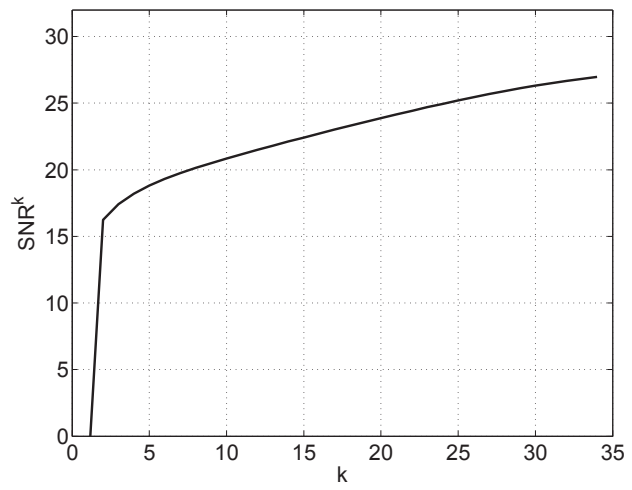

Fig. 6: The $\mathrm{SNR}^{k}$ after each iteration of the computation of $g_{k}$ with band-limit $L=17$ and spatial concentration in an elliptical region $\mathcal{R}_{\left(\theta_{\mathrm{c}}, a\right)}$ with focus $\theta_{\mathrm{c}}=\pi / 6$ and $a=\pi / 6+$ $\pi / 60$.

azimuthally symmetric and elliptical spatial regions. We have also carried out the numerical accuracy analysis of the proposed method and demonstrated that the sufficient accuracy is attained by the proposed method.

\section{REFERENCES}

[1] F. J. Simons, F. A. Dahlen, and M. A. Wieczorek, "Spatiospectral concentration on a sphere," SIAM Rev., vol. 48, no. 3, pp. 504-536, 2006.

[2] D. N. Spergel, R. Bean, O. Doré, M. R. Nolta, C. L. Bennett, J. Dunkley, G. Hinshaw, N. Jarosik, E. Komatsu, L. Page, H. V. Peiris, L. Verde, M. Halpern, R. S. Hill, A. Kogut, M. Limon, S. S. Meyer, N. Odegard, G. S. Tucker, J. L. Weiland, E. Wollack, and E. L. Wright, "Threeyear Wilkinson Microwave Anisotropy Probe (WMAP) observations: Implications for cosmology," The Astrophysical Journal Supplement Series, vol. 170, no. 2, pp. 377-408, 2007.

[3] D. W. Ritchie and G. J. L. Kemp, "Fast computation, rotation, and comparison of low resolution spherical harmonic molecular surfaces," J. Comput. Chem., vol. 20, no. 4, pp. 383-395, 1999.

[4] W. Zhang, M. Zhang, R. A. Kennedy, and T. D. Abhayapala, "On high-resolution head-related transfer function measurements: An efficient sampling scheme," IEEE Trans. Acoust., Speech, Signal Process., vol. 20, no. 2, pp. 575-584, 2012.

[5] T. S. Pollock, T. D. Abhayapala, and R. A. Kennedy, "Introducing space into MIMO capacity calculations," J. Telecommun. Syst., vol. 24, no. 2, pp. 415-436, Oct. 2003.

[6] D. Slepian and H. O. Pollak, "Prolate spheroidal wave functions, Fourier analysis and uncertainity-I," Bell Syst. Techn. J., vol. 40, pp. 43-63, Jan. 1961.

[7] H. J. Landau and H. O. Pollak, "Prolate spheroidal wave functions, Fourier analysis and uncertainity-II," Bell System Tech J., vol. 40, pp. 65-84, Jan. 1961.

[8] A. Albertella, F. Sansò, and N. Sneeuw, "Band-limited functions on a bounded spherical domain: the Slepian problem on the sphere," $J$. Geodesy, vol. 73, no. 9, pp. 436-447, Jun. 1999.

[9] M. A. Wieczorek and F. J. Simons, "Localized spectral analysis on the sphere," Geophys. J. Int., vol. 162, no. 3, pp. 655-675, Sep. 2005.

[10] - "Minimum variance multitaper spectral estimation on the sphere," J. Fourier Anal. Appl., vol. 13, no. 6, pp. 665-692, 2007.

[11] Z. Khalid, S. Durrani, P. Sadeghi, and R. A. Kennedy, "Spatio-spectral analysis on the sphere using spatially localized spherical harmonics transform," IEEE Trans. Signal Process., vol. 60, no. 3, pp. 1487-1492, Mar. 2012.

[12] — "Concentration uncertainty principles for signals on the unit sphere," in Proc. IEEE Int. Conf. Acoustics, Speech and Signal Processing, ICASSP'2012, Kyoto, Japan, March 2012, pp. 3717-3720.
[13] Z. Khalid, R. A. Kennedy, S. Durrani, P. Sadeghi, Y. Wiaux, and J. D. McEwen, "Fast directional spatially localized spherical harmonic transform," IEEE Trans. Signal Process., vol. 61, no. 9, pp. 2192-2203, 2013.

[14] Z. Khalid, R. A. Kennedy, and S. Durrani, "On the choice of window for spatial smoothing of spherical data," in Proc. IEEE Int. Conf. Acoustics, Speech and Signal Processing, ICASSP'2014, Florence, Italy, May 2014, pp. 2663-2667.

[15] R. A. Kennedy and P. Sadeghi, Hilbert Space Methods in Signal Processing. Cambridge, UK: Cambridge University Press, Mar. 2013.

[16] R. A. Kennedy, T. A. Lamahewa, and L. Wei, "On azimuthally symmetric 2-sphere convolution," Digital Signal Processing, vol. 5, no. 11, pp. 660-666, Sep. 2011.

[17] D. Coppersmith and S. Winograd, "Matrix multiplication via arithmetic progressions," in Proceedings of the Nineteenth Annual ACM Symposium on Theory of Computing, New York, NY, USA, 1987, pp. 1-6.

[18] Z. Khalid, R. A. Kennedy, and J. D. McEwen, "An optimaldimensionality sampling scheme on the sphere for fast spherical harmonic transforms," IEEE Trans. Signal Process., 2014, Accepted (available online)

[19] J. D. McEwen and Y. Wiaux, "A novel sampling theorem on the sphere," IEEE Trans. Signal Process., vol. 59, no. 12, pp. 5876-5887, Dec. 2011. 